\title{
RELIABILITY OF A SYSTEM OF THREE, FIRST ORDER ORDINARY DIFFERENTIAL EQUATION USING A SPARRE ANDERSEN PROCESS.
}

\section{USENI PAUL FATIYE ${ }^{1}$, SUNDAY SHAMMAH KPANJA ${ }^{1}$ AND PATRICIA NOKDET PAUL ${ }^{2}$}

\author{
${ }^{1}$ Department of Mathematics \& Statistics, Isa Mustapha Agwai I Polytechnic, Lafia, Nigeria. \\ ${ }^{2}$ Department of Computer Science, Isa Mustapha Agwai I Polytechnic, Lafia, Nigeria.; \\ Corresponding author: paul_useni@yahoo.com; +2348036161732 \\ DOI: $10.31364 /$ SCIRJ/v9.i05.2021.P0521860 \\ http://dx.doi.org/10.31364/SCIRJ/v9.i05.2021.P0521860
}

\begin{abstract}
In this paper, we discussed the existence of one explicit solution and apply a numerical method to test the reliability of a system of three, first order ordinary differential equations dependent on the choice of distribution function $p(x)$ The paper further shows how to determine the moments of the time to ruin in the Sparre Andersen risk model by evaluating and employing a function form for $\phi$. The work also discovered that the second order integro-differential equation might not have an explicit solution for a given probability function $p(x)$
\end{abstract}

Key Words: Sparre Andersen Model; reliability; numerical method; explicit solution; and probability function; integro-differential equation, ordinary differential equation.

\section{INTRODUCTION}

The Sparre Andersen risk process model was considered to recreate some of the studies in [1]. The claim inter-arrival distribution is Erlang $(2, \beta)$ used for analyzing the behavior of an explicit solution. This problem was studied before by [2] which considered the classic risk model. Modelling an insurance company who experiences two opposing cash flows, the incoming cash premiums and outgoing claims will allow us to evaluate expressions for moments of time to ruin, given that ruin occurs. Dickson and Hipp defined an auxiliary function along the lines of Hans Gerber and Elias Shin in [3] on which the moments of time to ruin are dependent.

In [9], a closed-form representation for the distribution of the ruin time for the Sparre Andersen model with exponentially distributed claim was studied while in [8], it was extended to the process with Erlang inter-claim times which was illustrated and the results in the cases of gamma and mixed exponential inter-claim time distribution was seen. Considering a risk process where the claims occur is distributed as Erlang. $(2, \beta)$, we assume that the time between claims (including the time until the first claim) form a sequence of independent and equal distributed random variables, denoted as $\left\{T_{i}\right\}_{j=1}^{\infty}$, with probability density function given by

$$
k(t)= \begin{cases}\beta^{2 t e-\beta t} & \text { t>0 } \\ 0 & \text {,elsewhere }\end{cases}
$$

The Sparre Andersen risk process defined as

$u+c t-\sum_{j=1}^{N_{t}} X_{j}$

Where $u \geq 0$ is the initial surplus, $c>0$ denotes the cash premium, which are received continuously at a constamt rate per unit time, $N_{t}$ is the mumber of claims up until time $t$ and $\left\{T_{i}\right\}_{j=1}^{\infty}$, are independent and identically distributed random variables, where $X_{j}$ is the amount of the $j^{\text {th }}$ claim. We define $p$ to be the distribution function of $X_{j}$ and then assume throughout that $X_{j}$ has a density function $p(x)$ [1]. Let $T=\inf \left\{t \mid U_{t}<0\right\}$ denote the time of ruin $(T=\infty$ if ruin does not occur). Then we consider the probability of ultimate ruin as a function of the initial surplus $U_{0}=u \geq 0$ [3],.

$$
\psi(u)=\operatorname{Pr}\left(T<\infty \mid U_{0}=0\right)
$$

Dickson and Hipp here introduced the Gerber-Shiu function to đefine $\phi$.

www.scirj.org

(C) 2021, Scientific Research Journal

http://dx.doi.org/10.31364/SCIRJ/v9.i05.2021.P0521860

This publication is licensed under Creative Commons Attribution CC BY. 


$$
\phi(u)=E\left[e^{-\delta T 1\{T<\infty\} \mid U_{0}=u}\right]
$$

Where $\delta$ can be interpreted as a force of interest and ${ }^{1}\{$.$\} is the usual indicator function [19]. Here we must note that if \delta=0, \phi(u)=$ $\psi(u)$. By Dickson and Hipp [1], we have

$$
\left.(-1)^{k} \frac{d^{k}}{d \delta^{k}} \phi(u)\right|_{\delta=0}=E\left[T^{k} 1\{T<\infty\} \mid U_{0}=u\right]
$$

And can use this to find moments of time to ruin.

\section{FORMULATION OF SECOND ORDER INTEGRO-DIFFERENTLAL EQUATION FOR $\phi$}

Given the Gerber-Shiu function (3) our immediate goal is to derive a functional equation for $\phi$. If we consider the time interval $(0, \infty)$, then we condition on the time $t$ and the amount $x$ of the first claim in this time interval [5] [7]. Note that the probability that there is no claim on $(0, \infty)$ is 0 and the probability that the first claim occurs on some interval $t, t+d t$ is $k(t) d t$ and $u+c t<x$ means that ruin has occurred with the first claim [3][6]. Thus conditioning on the time and the amount of the first claim, we are able to form the following equation

$\phi(u)=\int_{0}^{\infty} k(t) e^{-\delta t} \int_{0}^{u+c t} \phi(u+c f-x) p(x) d x d t+\int_{0}^{\infty} k(t) e^{-\delta t} \int_{u+c t}^{\infty} p(x) d x d t(5)$

Applying the change of variable to (5) yields

$\alpha \phi(u)=\int_{u}^{\infty} k\left(\frac{s-u}{c}\right) e^{-\delta(s-u) / c} \int_{0}^{s} \phi(s-x) p(x) d x d s+\int_{u}^{\infty} k\left(\frac{s-u}{c}\right) e^{-\delta(s-u) / c} \int_{s}^{s} p(x) d x d s(6)$

Applying a limit for the improper integral and then differentiating (6) gives

$$
\lim _{\gamma \rightarrow+\infty}\left(c \frac{d \phi}{d u}\right)=-\frac{1}{c}\left\{\int_{u}^{\infty} k^{\prime}\left(\frac{s-u}{e}\right) e^{-\delta(s-u) / c} \int_{s}^{\infty} \phi(s-u) p(x) d x d s+\int_{u}^{\infty} k^{\prime}\left(\frac{s-u}{e}\right) e^{-\delta(s-u) / c} \int_{s}^{\infty} p(x)\right\}
$$

Hence, substituting $k^{\prime}\left(\frac{s-u}{e}\right)=\beta^{2} e^{-\beta(s-u) / c}-\beta k\left(\frac{s-u}{c}\right)$ into (7) we obtain the following result (8)

Thus, we have the following first order integro-differential equation.

$c^{2} \frac{d \phi}{\mathrm{da}}-(\beta+\delta) c \phi(u)=-\int{ }_{u}^{\infty} \beta^{2} e^{-\frac{(\beta+\delta)(s-u)}{c}} \int{ }_{0}^{s} \phi(s-x) p(x) d x d s-\int{ }_{u}^{\infty} \beta^{2} e^{-\frac{(\beta+\delta)(s-u)}{c}} \int{ }_{s}^{\infty} p(x) d x d s$

Again, applying a limit for the improper integral and then differentiating

(9) we obtained the following result.

$$
\lim _{\gamma \rightarrow+\infty}\left[c^{2} \frac{d^{2} \phi}{d u^{2}}-(\beta+\delta) c \frac{d \phi}{d u}\right]=
$$

$(\beta+\delta) c \frac{d \phi}{d u}-(\beta+\delta)^{2} \phi(u)+\beta^{2} \int_{0}^{u} \phi(u-x) p(x) d x+\beta^{2}(1-p(u))$

Hence, we have successfully obtained the second order integro-differential equation satisfying $\phi$ as given below:

www.scirj.org

(C) 2021, Scientific Research Journal

http://dx.doi.org/10.31364/SCIRJ/v9.i05.2021.P0521XX

This publication is licensed under Creative Commons Attribution CC BY. 
$c^{2} \frac{d^{2} \phi}{d u^{2}}-2(\beta+\delta) c \frac{d \phi}{d u}+(\beta+\delta)^{2} \phi(u)=\beta^{2} \int_{0}^{u} \phi(u-x) p(x) d x+\beta^{2}(1-p(u))$

\section{LUNDBERG's FUNDAMENTAL EQUATION}

By the terminology of Gerber and Shiu [3], we define Lundberg's fundamental equation for the model [1].

$$
l(s)=c^{2} s^{2}-2(\beta+\delta) c s+(\beta+\delta)^{2}
$$

Where $\delta$ is strictly positive. By Dickson and Hipp [1], at $s=0$ we have

$$
l(0)=(\beta+\delta)^{2}>\beta^{2}=\beta^{2} p^{*}(0)
$$

Where $p^{*}(s)$ defines the Laplace transform of $p(t)$ [4]. Differentiating $i$, we have

$$
l^{\prime}(s)-2 c^{2} s-2(\beta+\delta) c
$$

Setting $l^{\prime}(s)=0$, we find that $l$ has a tuming point at

$$
s=\frac{\beta+\delta}{c}
$$

Further $l^{\prime}(s)=2 c^{2}>0$ hence $l(s)$ has its minimum at $s=\frac{\beta+\delta}{c}$. Now we may note that $l\left(\frac{\beta+\delta}{c}\right)=0, l(s)=\lim _{s \rightarrow \infty}=\infty$ and

$$
\frac{d}{d s} \beta^{2} p^{*}(s)=\beta^{2} \int_{0}^{\infty} x e^{-s x} p(x) d x<0
$$

so $\beta^{2} p^{*}(s)$ is a decreasing function of $s$ and is always positive. Hence,

$$
l(s)=\beta^{2} p^{*}(s)=0, s>0
$$

at two distinct points, one on each side of

$$
s=\frac{\beta+\delta}{c}
$$

\section{THE EXPLICIT SOLUTION $\phi(u)=\left(1-\frac{R}{\alpha}\right) e^{-R u}$}

Where Dickson and Hipp considered two individual claim amount distributions, both a mixture of two exponentials and a single exponential. we only consider the latter case. We will show how to find the moments of the time to ruin in the Sparre Andersen risk model by evaluating and employing a function form for $\phi[6]$.

$$
P(x)=1-e^{-\alpha x}, x>0
$$

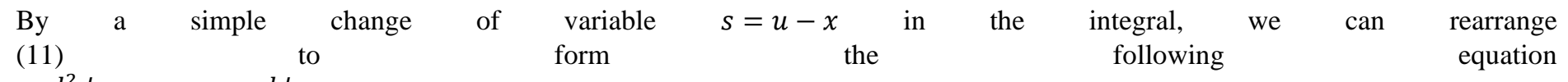
$c^{2} \frac{d^{2} \phi}{d u^{2}}-2(\beta+\delta) c \frac{d \phi}{d u}+(\beta+\delta)^{2} \phi(u)=\beta^{2} \int_{0}^{\infty} \phi(s) p(u-s) d s+\beta^{2}(1-p(u))$

Hence, for our form of $P(x)$, (15)becomes

www.scirj.org

(C) 2021, Scientific Research Journal

http://dx.doi.org/10.31364/SCIRJ/v9.i05.2021.P0521860

This publication is licensed under Creative Commons Attribution CC BY. 
$c^{2} \frac{d^{2} \phi}{d u^{2}}-2(\beta+\delta) c \frac{d \phi}{d u}+(\beta+\delta)^{2} \phi(u)=\beta^{2} e^{-\alpha x}\left[\alpha \int_{0}^{\infty} \phi(x) e^{\alpha x} d x+1\right]$

Differentiating (16), we shall have a 3rul order ordinary differential equation as follows:

$$
\begin{gathered}
c^{2} \frac{d^{2} \phi}{d u^{2}}-2(\beta+\delta) c \frac{d \phi}{d u}+(\beta+\delta)^{2} \phi(u)=\alpha \beta^{2} \int_{0}^{\infty} \phi(x) e^{-a(u-x)} d x+\beta^{2} e^{-\alpha v} \\
=-\alpha \beta^{2} e^{-\alpha x}\left[\alpha \int_{0}^{\alpha} \phi(x) e^{a x} d x+1\right] \\
c^{2} \frac{d^{3} \phi}{d u^{3}}+\left[\alpha c^{2}-2(\beta+\delta) c\right] \frac{d^{2} \phi}{d u^{2}}+\left[(\beta+\delta)^{2}-2 \alpha(\beta+\delta) c\right] \frac{d \phi}{d u}+\left[\alpha(\beta+\delta)^{2}-\alpha \beta^{2}\right] \phi(u)=0
\end{gathered}
$$

Given (14), we can calculate $p(x)=a e^{-\alpha x}$, since $p(x)$ is just the anti-derivative of $p(x)$. Substituting $p(x)$ into(13), we obtained the result with

$$
\begin{array}{r}
p^{*}(s)=\frac{\alpha}{s+\alpha} \\
c^{2} s^{2}-2(\beta+\delta) c s+(\beta+\delta)^{2}-\frac{\alpha \beta^{2}}{s+\alpha}=0 \\
\Rightarrow c^{2} s^{2}+\left[a c^{2}-2(\beta+\delta) c\right] s^{2}+\left[(\beta+\delta)^{2}-2 \alpha(\beta+\delta) c\right] s+\left[a(\beta+\delta)^{2}-\alpha \beta^{2}\right]=0
\end{array}
$$

Hence, the auxiliary equation of (17) has equal roots to the Lundeberg equation with substituted $p(x)$ into(18). These roots are $r_{1}, r_{2},-R$ and so we have the general solution for $\phi(u)$.

$\phi(u)=\eta_{1} e^{r_{1} u}+\eta_{2} e^{r_{2} u}+\eta_{3} e^{-R_{u}}$

Now as $\phi(u) \rightarrow 0$ as $u \rightarrow \infty$, we must have that the coeflicients $\eta_{1}=\eta_{2}=0$ resulting to

$$
\phi(u)=\phi(0) e^{-R u}
$$

After substituting (20) into (16), we have that

$$
\phi(0)=1-\frac{R}{\alpha}
$$

and thus, our explicit solution for $\phi$ is given by

$$
\phi(u)=\left(1-\frac{R}{\alpha}\right) e^{-R_{u}}
$$

4.1. A numerical method. For complex equation it can be somehow difficult to evaluate an analytical solution. However, it is possible that for the second order integro-differential equation (11), may not have an explicit solution for given probability function $P(x)$. In such situation, a numerical method should be employed to assess the behaviour of $\phi(u)$ as $u \rightarrow \infty$.

Though, it is simpler to apply a numerical method to an ordinary differential equation rather than an integro-differential equation. we can choose to employ numerical method to the third order ordinary differential equation ( 17) as it is equivalent to the second order integro-differential equation (11).

www.scirj.org

(C) 2021, Scientific Research Journal

http://dx.doi.org/10.31364/SCIRJ/v9.i05.2021.P0521XX

This publication is licensed under Creative Commons Attribution CC BY. 


\subsection{Third order ordinary differential equation}

The third order ordinary differential equation (11), can be reduced to s system of three first order, ordinary differential equations to enable us apply a numerical method. If we make the two substitutions,

$$
\omega(u)=\frac{d X}{d u}, X(u)=\frac{d \phi}{d u}
$$

Then, we are able to reduce (11) to the following system.

$$
\begin{gathered}
\frac{d \phi}{d u}=X(u) \\
\frac{d X}{d u}=\omega(u) \\
\frac{d \omega}{d u}=-\frac{1}{c^{2}}\left[\left(\alpha^{2} c^{2}-2(\beta+\delta) c\right) \omega(u)+\left((\beta+\delta)^{2}-2 \alpha(\beta+\delta) c\right) X(u)+\left(\alpha(\beta+\delta)^{2}-\alpha \beta^{2}\right) \phi(u)\right]
\end{gathered}
$$

\subsection{The explicit Euler method and the system of ordinary differential equations}

Using the explicit Euler method to (22), the system becomes;

$$
\begin{gathered}
\phi_{n+1}=\phi_{n}+h X_{n} \\
\phi_{n+1}=X_{n}+h \omega_{n} \\
\omega_{n+1}=\omega_{n}-\frac{h}{c^{2}}\left[\left(\alpha c^{2}-2(\beta+\delta) c\right) \omega_{n}+\left((\beta+\delta)^{2}-2 \alpha(\beta+\delta) c\right) X_{n}+\left(\alpha(\beta+\delta)^{2}-\alpha \beta^{2}\right) \phi_{n}\right]
\end{gathered}
$$

With initial conditions, $\phi o, X o$ and $\omega o$ and where $\phi_{\mathrm{n}}=\phi(n h), X_{n}=X(n h), \omega_{n}=\omega(n h)$ and $h$ is the step length.

4.2. Relationship of numerical and explicit solutions. Given an explicit solution (21), and the numerical method for the system of ordinary differential equations, graphs are drawn to see the relationship of the numerical solution with that of explicit solution.

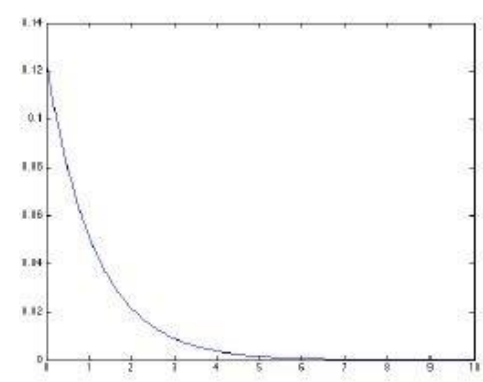

Figure1: Relationship of the numerical solution with initial conditions

$$
\phi_{1}=1-\frac{R}{\alpha}, X_{0}=R+\frac{R^{2}}{\alpha} \text { and } \omega_{0}=R^{2}-\frac{R^{3}}{\alpha}
$$

www.scirj.org

(C) 2021, Scientific Research Journal

http://dx.doi.org/10.31364/SCIRJ/v9.i05.2021.P0521860

This publication is licensed under Creative Commons Attribution CC BY. 


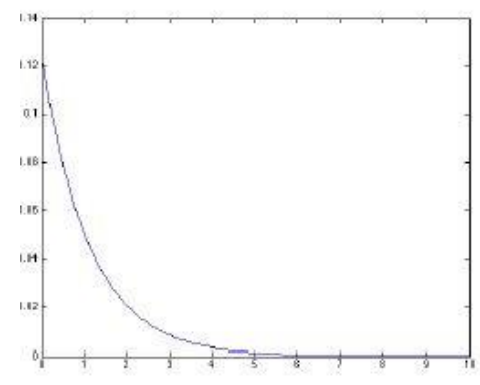

Figure 2: Relationship of the explicit solution.

\section{DISCUSSIONS}

We have seen that the relationship between numerical and explicit solutions in figures 1 and 2 respectively are similar when $\phi(u) \rightarrow$ 0 as $u \rightarrow \infty$. This gives a good indication that if we are unable to evaluate an explicit solution for a given probability function $p(x)$.

The chosen numerical method applied to the system of three, first order ordinary differential equations will be dependent on the choice of distribution function $p(x)$. We then know that we will be able to assess the ruin for other solutions and probability functions without need to find an explicit solution.

\section{CONCLUSION}

In conclusion, we have been able to see the reliability of a system of three. first order ordinary differential equations using Sparre Andersen process. Also, the moments of the time to ruin in the Sparre Andersen risk model by evaluating and employing a function form for $\phi$, for $p(x)=a e^{-\alpha x}$. Similarly, in a case where a second order integro-differential equation has no explicit solution for a given probability function $p(x)$, a numerical method should be employed to show the relationship between the numerical and explicit solutions which have proven that it only depends on the choice of distribution function of $p(x)$ as $u \rightarrow \infty$. which will result to having a similar relationship.

\section{Acknowledgement}

The authors are so grateful to the reviewers whose suggestions and corrections help to improve and add value to the paper, and also thankful to our Dean, School of Science and Technology, Mr. Solomon J. Anzene for his encouragement and support.

\section{REFERENCES}

[1] David C.M.Dickson and Christian Hipp, On the time to ruin for erlang(2) risk processes insurance, Mathematics and Economics, 29(3):333-344, December,2001.

[2] X,Sheldon Lin and Gordon E.Willnot, The moments of the time ruin, the surplus before ruin, and the deficit at ruin insurance, mathematics and Economics, 27 (1),19-44, August, 2000.

[3] Hans U.Gerber and Elias S.W.Shiu, On the time value of ruin. , North American Actuarial Journal,2(1):48-72; 1998.

[4] Macro Taboga, Lectures on probability theory and mathematical statistics, Amazon Create Space, second edition, December,2012

[5] Paul Dachateau and D.W. Zachmann, Schaum's outline of partial Differential Equations, Schaum's outline series, Schaum's outlines, revised edition, March; 2011

[6] Frank P. Incopera, David P. DeWitt, Theodore L.Bergman, and Adrienne S. Lavine, Fundamentals of heat and mass transfer. , John Wiley \& Sons sixth edition, December,2001

[7] Konstantin A.Borovkov A. and David C. M. Dickson, On the ruin time distribution for a Sparre Andersen process with exponential claim sizes, Centre for Actuarial Studies, the University of Mebourne, September, 2007.

[8] D.C.M Dickson; B.D. hughes and L.Zhang, The density of the time to ruin for a Sparre Andersen process with Erlang arrivals and exponential claims, Scand, Actuarial Journal,358-376,2005

www.scirj.org

(C) 2021, Scientific Research Journal

http://dx.doi.org/10.31364/SCIRJ/v9.i05.2021.P0521XX

This publication is licensed under Creative Commons Attribution CC BY. 
